\title{
A novel method for internal fixation of basal fifth metatarsal fracture in athletes: a cadaveric study of the F.E.R.I. technique (Fifth metatarsal, Extra-portal, Rigid, Innovative)
}

Pieter D'Hooghe ${ }^{1}$, Silvio Caravelli $i^{*}$ D, Simone Massimi ${ }^{2}$, James Calder ${ }^{3,4}$, Peter Dzendrowskyj ${ }^{1}$ and Stefano Zaffagnini ${ }^{2}$

\begin{abstract}
Purpose: One of the main problems of Kirschner wire fixation of fifth metatarsal base fractures (in combination with a tension band wiring technique) seems to be hardware intolerance and several studies in athletes also report failure after isolated fixation with a screw only. These reports prompted us to look at new materials and a novel technique through fixation with an intramedullary screw combined with a high-resistance suture via the presented F.E.R.I. (Fifth metatarsal, Extra-portal, Rigid, Innovative) technique.

Methods: This cadaveric study describes F.E.R.I. technique. On a cadaver, through two mini portals, a full reduction and solid internal fixation with an intramedullary screw and suture cerclage with Fiberwire of a fifth metatarsal base fracture is achieved. In this article, the cadaveric study and proposed surgical technique are explained and illustrated step by step.

Results: The presented internal fixation F.E.R.I. technique is indicated in acute proximal fractures, stress fractures or non-union of metatarsal 5 (Zone 2-3 by Lawrence and Botte) and it resulted feasible and stable during manual stress test. The authors intend to study this technique in the clinical setting in the near future.

Conclusions: Fifth metatarsal base fractures gain specific interest when occurring in athletes. In this group of patients, internal fixation is often required to obtain a satisfactory outcome and time to return to play. The aim of the presented cadaveric study is to illustrate an innovative concept of internal fixation, named F.E.R.I.
\end{abstract}

Keywords: Fifth metatarsal, Fracture, Internal fixation, Athlete

\section{Introduction}

Sir Robert Jones (Jones 1902), in 1902, first described fractures at the metaphyseal/diaphyseal junction of the fifth metatarsal (MT-5). These make up approximately $70 \%$ of all metatarsal fractures (Petrisor et al. 2006), both in the general population and also in athletes. In an

\footnotetext{
*Correspondence: doct.car@gmail.com

Investigations have been performed at Aspetar Orthopaedic and Sports Medicine Hospital - Doha (Qatar)

${ }^{2}$ 2nd Clinic of Ortopaedics and Traumatology, IRCCS Istituto Ortopedico

Rizzoli, Bologna, Italy

Full list of author information is available at the end of the article
}

interesting review, Spang RC et al (Spang et al. 2018) have found that the overall percentage of fifth metatarsal base fractures in NFL players was 3.2\%. Fifth metatarsal fractures are an increasing problem, particularly in athletes. Surgical treatment strategies and outcomes are, therefore, of particular interest among those involved in their care.

Orendurff et al.(Orendurff et al. 2009) demonstrated that during sport activities, bending moments and peak pressure are considerable through the fifth metatarsal. They highlighted how different athletic gestures, such as jump-off, landing and cutting movements can produce 
high tensile stress on bone structures - a forceful pushoff of acceleration generates a high load on the forefoot, particularly on the fifth metatarsal (Orendurff et al. 2009). This can lead to either acute fractures of the base of the fifth metatarsal or, during high periods of activity, to stress fractures.

Pre-disposing factors include: cavo-varus foot, prominence of the fifth metatarsus, high body mass index (BMI), and the use of narrow-width shoes (Jastifer et al. 2017; McBryde Jr. 2009). An indirect force secondary to foot plantar-flexion and inversion - transmitted to the MT-5 by the peroneus brevis and plantar fascia traction (Mayer et al. 2014; Glasgow and Naranja Jr 1997) - is the usual mechanism of injury leading to this proximal fracture. Increased forces during physical activity make it easy to understand how athletes, in particular are predisposed to this type of injury.

MT-5 fractures have been classified by Lawrence and Botte (Lawrence and Botte 1993) according to the anatomical area affected, and also the mechanism of injury, in Zone 1 (tuberosity area), Zone 2 (tuberosity - metaphyseal area) and Zone 3 (metaphyseal - diaphyseal area). Fractures in Zones 2 and 3 are commonly referred to as Jones' fractures, due to their similarity in symptoms, treatment and possible complications. Zones 2 and 3 are predisposed to delayed healing due to a vascular watershed zone (Smith et al. 1992; Le and Anderson 2017) between the insertion of peroneus brevis and the diaphyseal blood supply. Fractures interrupt the vascular channels in this area, leading to hypo-vascularisation and difficulty in complete healing.

Fractures of the proximal MT-5 can be treated either conservatively or surgically; Josefsson (Josefsson et al. 1994) demonstrated good functional outcomes with non-operative treatment. However, in athletes, treatment is usually surgical, because of the long recovery time, the higher risk of non-union and high rates of re-fracture associated with conservative treatment (Clapper et al. 1995; Kavanaugh et al. 1978; Raikin et al. 2008).

Several studies, however, report failure after fixation with screw, in particular, in athletes. Wright R. et al. (Wright et al. 2000) found that failure seemed to be correlated with the dimension rather than the type of screw (cannulated, cancellous or malleolar screws). He reported a $5 \%$ failure rate - a combination of refracture and nonunion. Granata JD. et al. (Granata et al. 2015) reported a $7.3 \%$ failure rate in patients with primary surgical treatment - mainly due to refracture. Because of this, other fixation methods have been developed aiming for better primary stability and a lower rate of complications and refractures. Internal fixation by tension band wiring has been used with similar outcomes (Delee et al. 1983; Lee et al. 2011). There have been many publications regarding fractures of the base of fifth metatarsal, however disagreement and controversy remain around the most appropriate surgical treatment.

This report describes and discusses an innovative concept for surgical fixation via a combination of screw and suture cerclage of MT-5 fractures in athletes, named F.E.R.I. "Fifth metatarsal, Extra-portal, Rigid, Innovative".

\section{Materials and methods}

The "two portal technique" (TPT), or "F.E.R.I. technique", is indicated for internal fixation of acute proximal fractures - or stress fractures of MT-5 - (Zone 2-3 by Lawrence and Botte) (Fig. 1). A further indication is chronic delayed union or non-union of proximal MT-5 fractures.

The feasibility of the technique has been tested on a cadaveric specimen. After creating a fifth metatarsal base fracture with a $10 \mathrm{~mm}$ osteotome, reduction and fixation has been performed under direct visual control.

\section{Surgical technique}

A $1 \mathrm{~cm}$ longitudinal stab incision ("proximal portal") is made $15 \mathrm{~mm}$ proximal to the base of the fifth metatarsal.

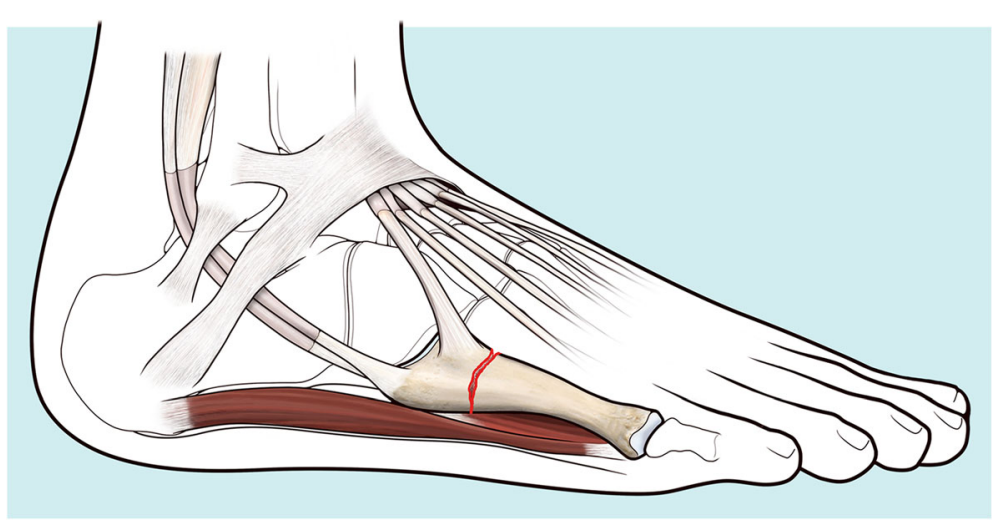

Fig. 1 Proximal fractures or stress fractures of MT-5 (Zone 2-3 by Lawrence and Botte) can be treated with the F.E.R.I. technique 
This reveals the insertion of the peroneus brevis tendon. Dissection identifies the lateral dorsal cutaneous nerve and its distal branches.

A second $15 \mathrm{~mm}$ incision ("distal portal") is made obliquely, at the same level as the fracture site, following Langer's skin folds, from dorsal to plantar and from proximal to distal (Fig. 2). Blunt subcutaneous dissection identifies the fracture line, which is then inspected and minimally debrided.

Under X-ray C-arm guidance, any displaced fracture is reduced. If a cannulated screw is used, a guide wire is then introduced through the proximal portal into the fifth metatarsal medullary cavity in a dorso-medial entry angle, medial to the tip of the base. This maximises screw length whilst protecting the insertion point of peroneus brevis (Fig. 3). A through-hole is drilled vertically through the distal port, $10 \mathrm{~mm}$ distal to the fracture line. An Arthrex Fiberwire ${ }^{\circ}$ (Arthrex, Naples, FL USA) no. 2 is passed through the hole (Fig. 2 and Fig. 4).

A $5.5 \mathrm{~mm}$ partially threaded screw is placed along the guide wire, if cannulated, and inserted into the medullary canal to across the fracture line (Fig. 3). After marking the dorsal end the suture wire with a marking pen, the Fiberwire is then pulled subcutaneously from the distal incision to the proximal one in a figure-of-eight pattern and looped around the neck of the screw (Fig. 5). The screw is then tightened and the Fiberwire cerclage properly tensioned and securely knotted (Fig. 6 and Fig. 7). Manual tests and maneuvers have been performed and fracture stability was achieved with success.

\section{Additional procedures}

In cases of delayed union, or to improve fracture consolidation, a small amount of autogenous cancellous bone can be harvested from superolateral aspect of the calcaneus.

A $10 \mathrm{~mm}$ "extra-portal" is made posteriorily and slightly distal to the Sural nerve and peroneal tendon. Subcutaneous blunt dissection is performed to reveal periosteum, which is then reflected back with a periosteum elevator. The bone graft borders are visualised, and

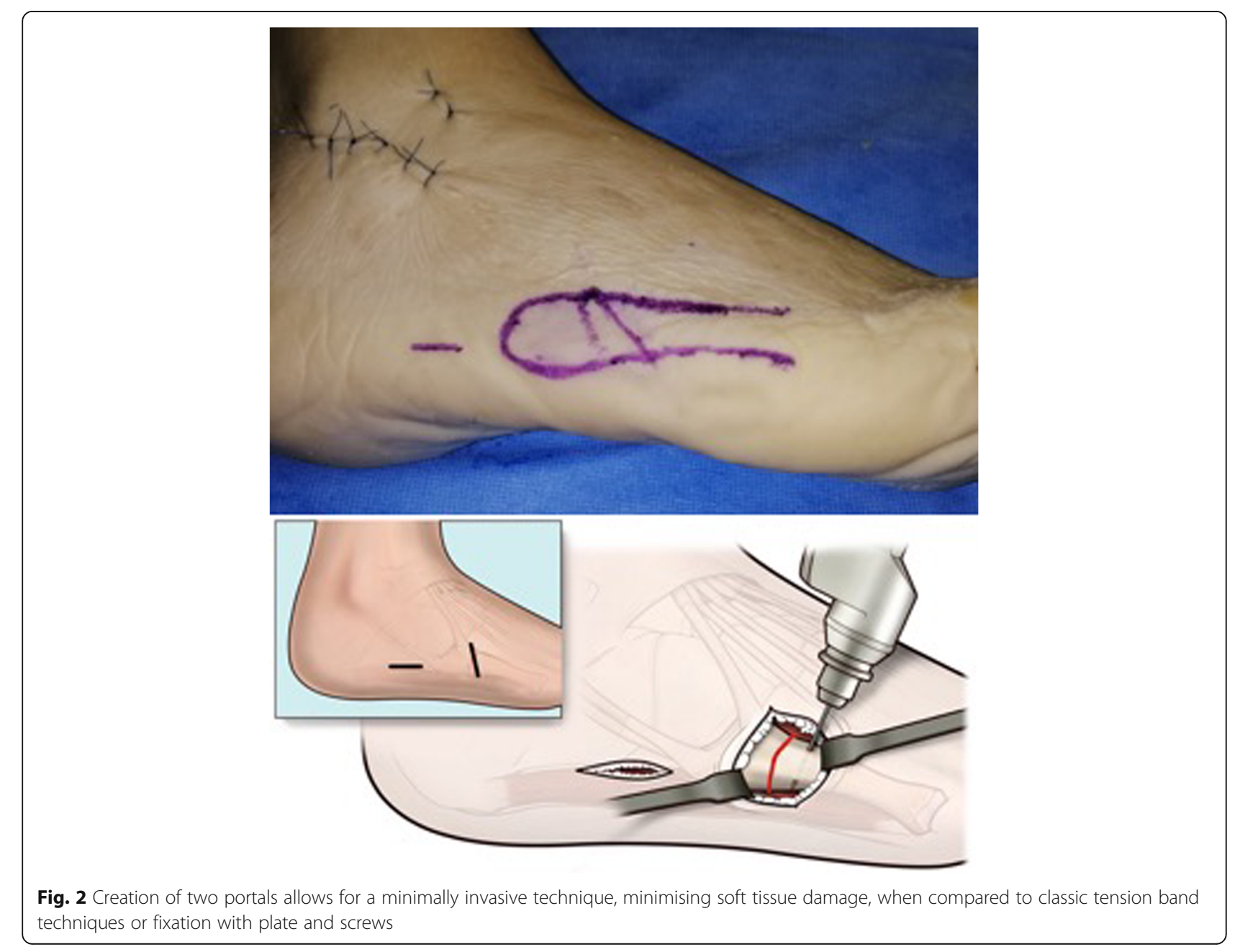




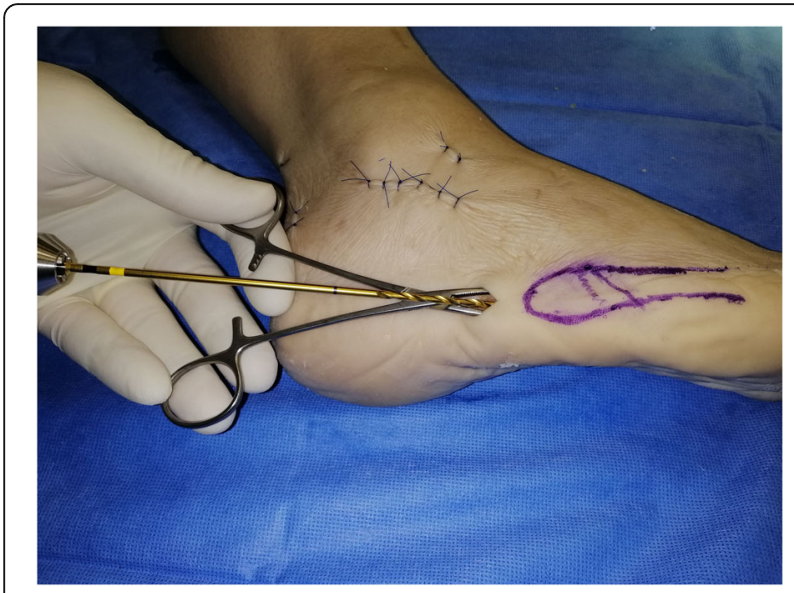

Fig. 3 Preparation for screw fixation. A hole for the screw is drilled at the entry point. A $5.5 \mathrm{~mm}$ partially threaded screw is placed in the medullary cavity through the entry point and screwed to, or just beyond, the fracture line

a carefully sized cortico-cancellous bone wedge can be harvested, using a small osteotome or a small oscillating saw.

Fluoroscopy can be used to identify the correct harvesting site. The cortico-cancellous autograft is placed like a wedge, with the cortical side positioned plantar,

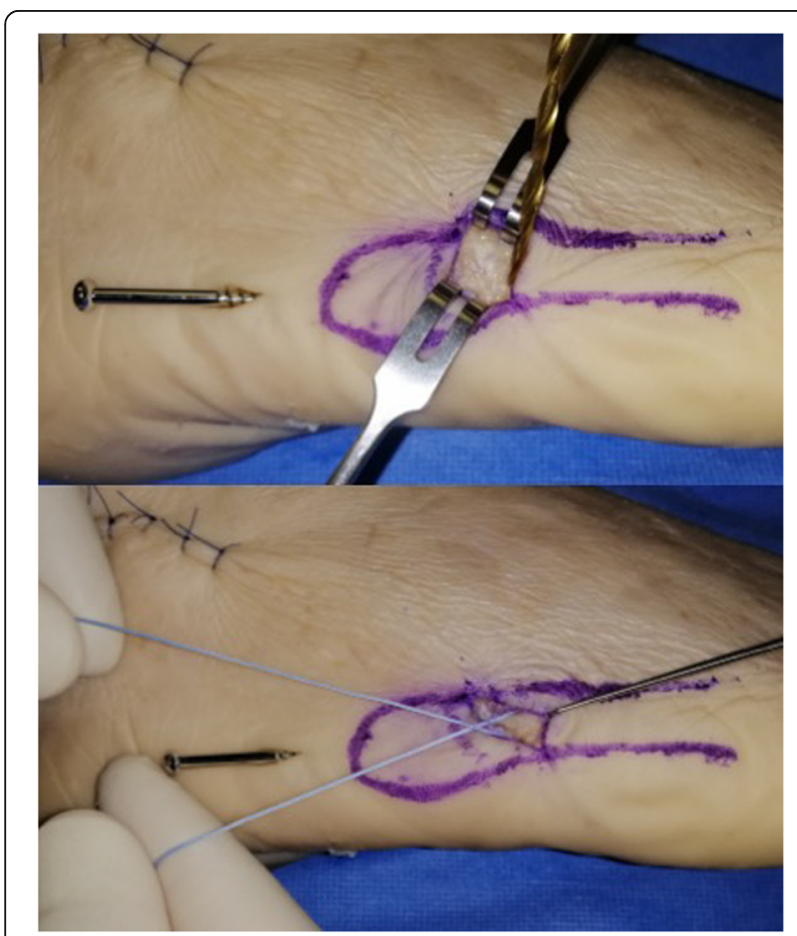

Fig. 4 Preparation of cerclage. A through-hole is drilled vertically through the distal port, $10 \mathrm{~mm}$ distal to the fracture line. An Arthrex Fiberwire $^{\circledast}$ (Arthrex, Naples, FL USA) no. 2 is passed through the hole using a figure-of-eight pattern

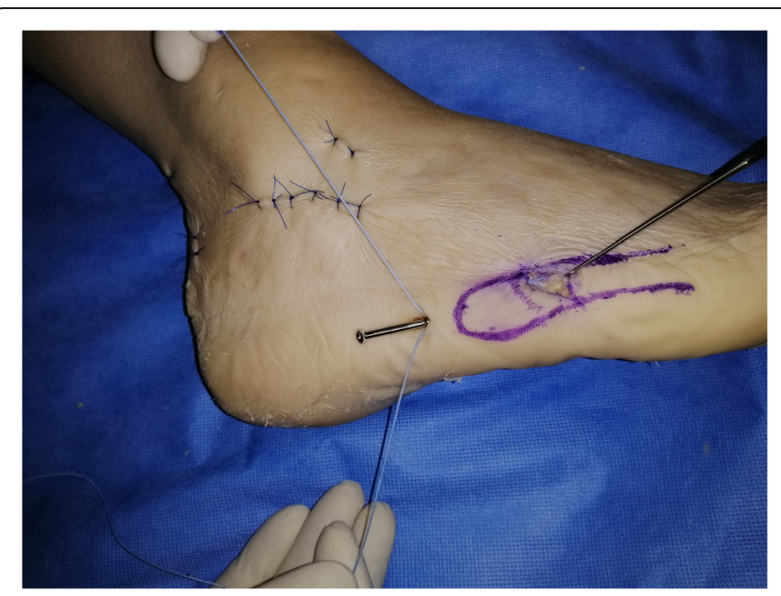

Fig. 5 The Fiberwire ${ }^{\varpi}$ is crossed subcutaneously, using small curved Klemmer forceps, from distal to proximal in a figure-of-eight pattern and looped around the neck of the screw. The dorsal wire is pulled plantarly and the plantar wire dorsally

before suture cerclage and fracture compression. Positioning is aimed at the crossing point of the figure-ofeight cerclage, which can then support the wedge.

\section{Discussion}

There have been many publications regarding fractures of the base of fifth metatarsal, however disagreement and controversy remain around the most appropriate surgical treatment.

Various techniques are available (Tsukada et al. 2012; Glasgow and Naranja Jr 1997; Wright et al. 2000) - the most popular being fixation by cannulated screw for proximal fractures of the fifth metatarsus (Kavanaugh et al. 1978; Lareau and Anderson 2015). Inserting a straight screw into the curved proximal fifth metatarsus can be a real challenge. The entry point for insertion

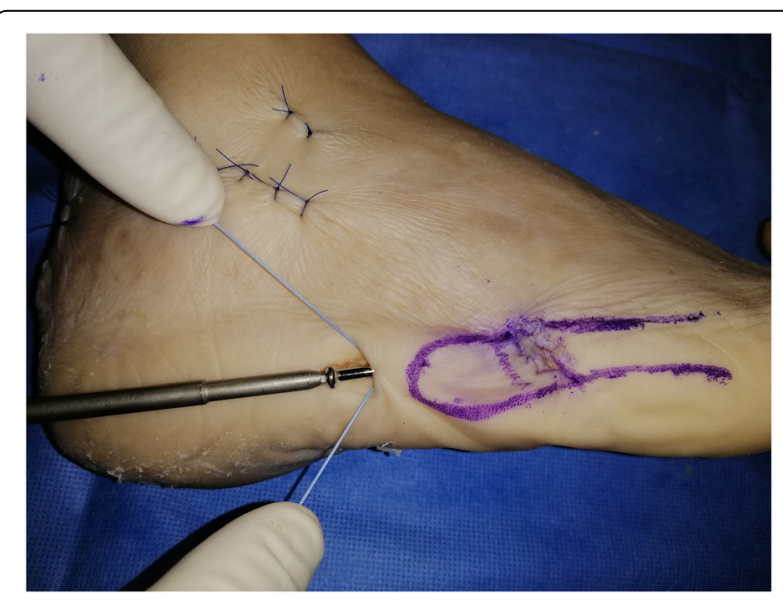

Fig. 6 The screw is then tightened, the Fiberwire ${ }^{\oplus}$ cerclage is properly tensioned and securely knotted 

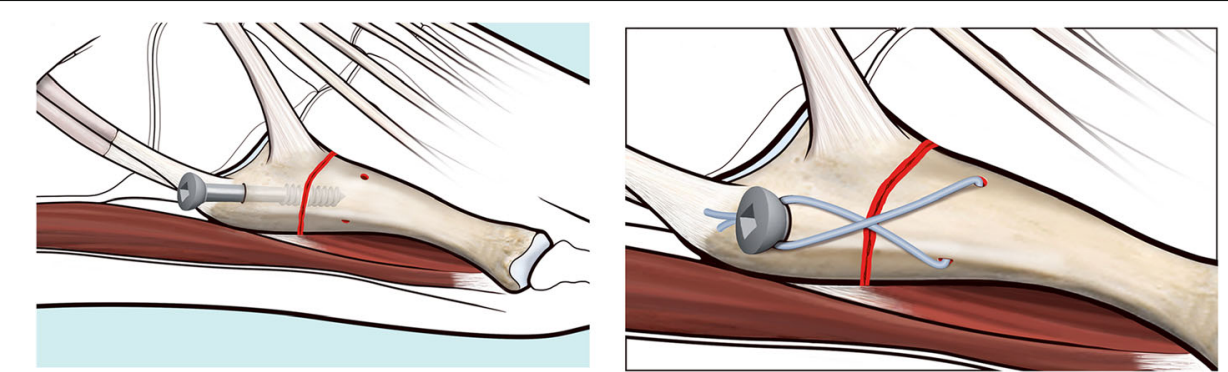

Fig. 7 Diagram of screw position, of cerclage hole and final result. Note that the screw entry point in the figure has been deliberately drawn slightly lateral to aid visualisation

needs to be immediately medial to the insertion fibres of peroneus brevis, avoiding branches of the Sural nerve (Donley et al. 1999; Fansa et al. 2012). Direction has to be $7^{\circ}$ from cranial to caudal - relative to the sole of the foot - pointing in the direction of the diaphysis of the metatarsus.

Recently, several biomechanical studies have been carried out to evaluate the ideal characteristics of the screw to obtain a good fixation and grip (Duplantier et al. 2018; Huh et al. 2016; Scott et al. 2015; Nagao et al. 2012; Porter et al. 2009). The diameter of the screw should be no less than $4.5 \mathrm{~mm}$ in order for there to be adequate compression across the fracture line. In general, the wire should be between 4.5 and $5.5 \mathrm{~mm}$ in diameter- in men the diameter of the 5th metatarsus is greater than in women. There is no significant difference in the strength of fracture fixation between these different diameter screws $(332.4 \mathrm{~N}$ versus $335.2 \mathrm{~N}$ between 4.5 and 5.5 screw) (Raikin et al. 2008).

Stainless steel cerclage is a type of internal fixation using the same concept as dynamic compression tension banding - tensile forces of bone eccentric loading are converted into compressive forces. Tension band fixation has undergone several modifications, since its introduction by Pauwels in 1980 (Pauwels 1980), ranging from the two-screws technique to the classical two Kirschner wires technique (Lee et al. 2011; Sarimo et al. 2006). Good results after tension band wiring fixation have been reported in the literature - it has become a well-documented procedure for the treatment of acute fifth metatarsal fractures and stress fractures (Sarimo et al. 2006; Nolte et al. 2016).

One of the main problems of fixation with Kirschner wires and tension band wiring, seems to be that of hardware intolerance, particularly in athletes. Several authors have reported the need to remove the orthopedic hardware due to this problem (Bryant et al. 2018; Curtis et al. 2015; Hasselman et al. 2003).

The classical technique involves two Kirschner wires inserted longitudinally in the metatarsal bone from the entry point at the medial base. Mofidi et al. (Mofidi et al. 2009), reported a case where a stress fracture of the fifth metatarsal was caused by tension band wiring itself - a known, though rare, complication. They describe a 26-year-old athlete who suffered a stress fracture 1 year after surgery, surmising that the loss of bone strength was due to both the presence in the medial cortex of the K-wire, as well as the hypo-vascularization of the bone segment.

These problems prompted us to look at new materials and novel techniques for the fixation of fractures at the base of the fifth metatarsal - particularly in athletes, who require greater stability for good functional recovery.

Stainless steel monofilament wire is strong and manageable and is still the material of choice for tension banding fixation in general traumatology practice. In recent years, many studies have compared other different synthetic suture materials (Flanigan et al. 2011; Mahar et al. 2006; Kuruvalli et al. 2008; Renner et al. 2014). A recent study by Westberg et al. (Westberg et al. 2017) compared the biomechanical characteristics of different sutures, including Fiberwire ${ }^{\circ}$ (Arthrex, Naples, FL USA) and a classic $1.2 \mathrm{~mm}$ stainless steel monofilament, evaluating their cyclic load and load to failure. The authors highlighted that the Fiberwire suture no. 2 had the highest tensile strength and load to failure of all materials in the study while the stainless steel monofilament had the lowest.

We suggest the use of both an intramedullary screw and a Fiberwire suture no. 2 cerclage together, combining the advantages of both techniques and reducing the possibility of failure. In patients with high functional demands, using an isolated intramedullary screw has been shown to be associated with poor resistance to dorsal flexion forces (Kavanaugh et al. 1978; Tsukada et al. 2012). This has resulted in either a greater risk of hardware rupture or insufficient stability of the fracture site. Stainless steel cerclage by itself can also fail if subjected to high load cycles, or can cause discomfort due to irritation of surrounding soft tissues. 
The creation of two ports allows for this technique to be minimally invasive, useful for preserving the vascularity of the area, which is already somewhat hypovascularised. Minimal periosteal removal is likely to reduce the risk of delayed healing or non-union by protecting soft tissue coverage, when compared to other more aggressive internal fixation methods.

This technique has not yet been used clinically and is - at present - hypothetical in nature. Proof of concept has, however, been shown to be effective - it has been performed on a cadaveric specimen, illustrating surgical feasibility and technical reproducibility, but it has been not biomechanically tested and its superiority compared to standard techniques has to be still proved.

A second limitation of this technique is that it is not directed at the general population, but aimed at professional and elite athletes. This is because of their greater functional requirement and greater biomechanical stress on the fracture, with the possibility of failure of fixation if carried out with intramedullary screw only. Therefore, it makes sense to have a more stable synthesis, using a combination of the two techniques.

Fixation with an intramedullary screw combined with a Fiberwire suture no. 2 via the F.E.R.I. technique, is a valid treatment option for acute fractures and stress fractures of the fifth base in athletes. Furthermore, this technique could be used in cases of delayed consolidation or non-union in which a biological augmentation is requested. The authors intend to study this technique in the clinical setting in the near future.

\section{Acknowledgments}

Authors would like to thank Dr. Maria Pia Cumani from Scuola di Disegno Anatomico, Rizzoli Orthopaedic Institute (Bologna, Italy) for the artwork and figures.

\section{Authors' contribution}

DHP conceived of the study, defined the intellectual contents and performed the cadaveric tests. SC performed the cadaveric tests and the literature search and drafted the manuscript. SM performed the cadaveric tests and the literature search and drafted the manuscript. JC performed the manuscript review and editing. PD performed the manuscript review and editing. SZ conceived of the study and participated in its design and coordination. All authors read and approved the final manuscript.

\section{Funding}

None.

\section{Competing interests}

The authors declare that they have no competing interests.

\footnotetext{
Author details

${ }^{1}$ Department of Orthopaedic Surgery and Sports Medicine, Aspetar Hospital, Doha, Qatar. ${ }^{2}$ 2nd Clinic of Ortopaedics and Traumatology, IRCCS Istituto Ortopedico Rizzoli, Bologna, Italy. ${ }^{3}$ Department of Orthopaedic Surgery, Fortius Clinic, London, UK. ${ }^{4}$ Department of Bioengineering, Imperial College London, London, UK.
}

Received: 29 August 2019 Accepted: 28 October 2019

Published online: 11 November 2019

\section{References}

Bryant T, Beck DM, Daniel JN et al (2018) Union rate and rate of hardware removal following plate fixation of metatarsal shaft and neck fractures. Foot Ankle Int 39(9):326-331

Clapper M, O'Brien T, Lyons P (1995) Fractures of the fifth metatarsal: analysis of a fracture registry. Clin Orthop Relat Res 315:238-241

Curtis BD, Fajolu O, Ruff ME et al (2015) Fixation of metacarpal shaft fractures: biomechanical comparison of intramedullary nail crossed K-wires and platescrew construct. Orthop Surg 7(3):256-260

Delee J, Evans J, Julian J (1983) Stress fracture of the fifth metatarsal. Am J Sports Med 11:349-353

Donley BG, McCollum MJ, Murphy GA et al (1999) Risk of sural nerve injury with intramedullary screw fixation of fifth metatarsal fractures: a cadaver study. Foot Ankle Int 20(3):182-184

Duplantier NL, Mitchell RJ, Zambrano S et al (2018) A biomechanical comparison of fifth metatarsal Jones fracture fixation methods. Am J Sports Med 46(5): $1220-1227$

Fansa AM, Smyth NA, Murawski CD et al (2012) The lateral dorsal cutaneous branch of the sural nerve: clinical importance of the surgical approach to proximal fifth metatarsal fracture fixation. Am J Sports Med 40(8):1895-1898

Flanigan DC, Bloomfield M, Koh J (2011) A biomechanical comparison of patellar tendon repair materials in a bovine model. Orthopaedics 34:344-348

Glasgow M, Naranja R Jr (1997) Analysis of failed surgical management of fractures of the base of the fifth metatarsal distal to the tuberosity: the Jones fracture. J Pediatr Orthop 17:277-278

Granata JD, Berlet GC, Philbin TM et al (2015) Failed surgical Management of Acute Proximal Fifth Metatarsal (Jones) fractures: a retrospective case series and literature review. Foot Ankle Spec 8(6):454-459

Hasselman CT, Vogt MT, Stone KL et al (2003) Foot and ankle fractures in elderly white women. Incidence and risk factors. J Bone Joint Surg Am 85(5):820-824

Huh J, Glisson RR, Matsumoto T et al (2016) Biomechanical comparison of intramedullary screw versus low-profile plate fixation of a Jones fracture. Foot Ankle Int 37(4):411-418

Jastifer J, Kent R, Crandall J et al (2017) The athletic shoe in football: apparel or protective equipment? Sports Health 9(2):126-131

Jones RI (1902) Fracture of the base of the fifth metatarsal bone by indirect violence. Ann Surg 35(6):697-700.2

Josefsson PO, Karlsson M, Redlund-Johnell I et al (1994) Closed treatment of Jones fracture. Good results in 40 cases after $11-26$ years. Acta Orthop Scand 65(5):545-547

Kavanaugh JH, Brower TD, Mann RV (1978) The Jones fracture revisited. JBJS 60(6):776-782

Kuruvalli RR, Landsmeer R, Debnath UK et al (2008) A new technique to reattach an extended tronchanteric osteotomy in revision THA using suture cord. Clin Orthop Relat Res 466:1444-1448

Lareau CR, Anderson RB (2015) Jones fractures pathophysiology and treatment. JBJS Rev 3(7):e4

Lawrence SJ, Botte MJ (1993) Jones' fractures and related fractures of the proximal fifth metatarsal. Foot Ankle 14(6):358-365

Le M, Anderson R (2017) Zone II and III fifth metatarsal fractures in athletes. Curr Rev Musculoskelet Med 10(1):86-93

Lee KT, Park YU, Young KW et al (2011) Surgical results of $5^{\text {th }}$ metatarsal stress fracture using modified tension band wiring. Knee Surg Sports Traumatol Arthrosc 19:853-857

Mahar AT, Moezzi DM, Serra-Hsu F et al (2006) Comparison and performance characteristics of three different knots when tied with two suture materials used for shoulder arthroscopy. Arthroscopy 22:610-614

Mayer SW, Joyner PW, Almekinders LC et al (2014) Stress fractures of the foot and ankle in athletes. Sports Health 6(6):481-491

McBryde AM Jr (2009) The complicated Jones fracture, including revision and malalignment. Foot Ankle Clin 14(2):151-168

Mofidi A, Hamer P, Thomas RH et al (2009) Stress fracture of the fifth metatarsa base caused by tension band wiring. An isolated case report. Foot Ankle Spec 2(2):79-82

Nagao M, Saita Y, Kameda S et al (2012) Headless compression screw fixation of Jones fractures: an outcomes study in Japanese athletes. Am J Sports Med 40(11):2578-2582 
Nolte P, Anderson R, Strauss E et al (2016) Heal rate of metatarsal fractures: a propensity-matching study of patients treated with low-intensity pulsed ultrasound (LIPUS) vs. surgical and other treatments. Injury, Int J Care Injured 47(11):2584-2590

Orendurff MS, Rohr ES, Segal AD et al (2009) Biomechanical analysis of stresses to the fifth metatarsal bone during sports maneuvers: implications for fifth metatarsal fractures. Physician Sports Med 2(37):87-92

Pauwels F (1980) Biomechanics of the locomotor apparatus, 1st edn. SpringerVerlag, Berlin Heidelberg New York

Petrisor BA, Ekrol I, Court-Brown C (2006) The epidemiology of metatarsal fractures. Foot Ankle Int 27(3):172-174

Porter DA, Rund AM, Dobslaw R et al (2009) Comparison of 4.5- and 5.5-mm cannulated stainless steel screws for fifth metatarsal Jones fracture fixation. Foot Ankle Int 30:27-33

Raikin SM, Slenker N, Ratigan B (2008) The association of a varus hindfoot and fracture of the fifth metatarsal metaphysealdiaphyseal junction: the Jones fracture. Am J Sports Med 36(7):1367-1372

Renner N, Wieser K, Lajtai G et al (2014) Stainless steel wire versus Fiberwire suture cerclage fixation to stabilize the humerus in total shoulder arthroplasty. J Shoulder Elb Surg 23:1568-1574

Sarimo J, Rantanen J, Orava S et al (2006) Tension-band wiring for fractures of the fifth metatarsal located in the junction of the proximal metaphysis and diaphysis. Am J Sports Med 34(3):476-480

Scott RT, Hyer CF, DeMill SL (2015) Screw fixation diameter for fifth metatarsal jones fracture: a cadaveric study. J Foot Ankle Surg 54(2):227-229

Smith JW, Arnoczky SP, Hersh A (1992) The intraosseous blood supply of the fifth metatarsal: implications for proximal fracture healing. Foot Ankle 13(3):143-152

Spang RC, Haber DB, Beaulieu-Jones BR et al (2018) Jones fractures identified at the National Football League Scouting Combine: assessment of prognostic factors, computed tomography findings, and initial career performance. Orthop J Sports Med 6(8):2325967118790740

Tsukada S, Ikeda H, Seki Y (2012) Intramedullary screw fixation with bone autografting to treat proximal fifth metatarsal metaphyseal-diaphyseal fracture in athletes: a case series. Sports Med Arthrosc Rehabil Ther Technol 4(1):25

Westberg SE, Acklin YP, Hoxha S et al (2017) Is suture comparable to wire for cerclage fixation? A biomechanical analysis. Should Elb 0(0):1-8

Wright R, Fischer D, Shively R et al (2000) Refracture of proximal fifth metatarsal (Jones) fracture after intramedullary screw fixation in athletes. Am J Sports Med 28:732-736

\section{Publisher's Note}

Springer Nature remains neutral with regard to jurisdictional claims in published maps and institutional affiliations.

\section{Submit your manuscript to a SpringerOpen ${ }^{\circ}$ journal and benefit from:}

- Convenient online submission

- Rigorous peer review

- Open access: articles freely available online

- High visibility within the field

- Retaining the copyright to your article

Submit your next manuscript at $\boldsymbol{\nabla}$ springeropen.com 